\title{
INDICADORES BIBLIOMÉTRICOS DOS ARTIGOS SOBRE "SEXUALIDADE NA ADOLESCÊNCIA" NA BASE SCOPUS
}

\author{
BIBLIOMETRIC INDICATORS OF ARTICLES ON "SEXUALITY IN ADOLESCENCE" \\ IN THE SCOPUS BASE
}

\author{
Leandra Ferreira Cardoso da Silva ${ }^{1}$, Flávio Rodrigues Ribeiro², Leonardo Muniz Soares Dias Duarte ${ }^{2}$, Shaytner \\ Campos Duarte ${ }^{2}$, Eduardo Shimoda ${ }^{1}$, Ronaldo Caetano Barboza ${ }^{3}$ \\ ${ }^{1}$ Universidade Candido Mendes - Campos \\ ${ }^{2}$ Faculdade de Medicina de Campos \\ ${ }^{3}$ Instituto Federal de Educação, Ciência e Tecnologia Fluminense - IF Fluminense
}

\section{RESUMO}

A sexualidade na adolescência é um problema que tem sido estudado com frequência em função do risco de gravidez indesejada e/ou não planejada, doenças sexualmente transmissíveis e vitimização sexual. O objetivo do presente trabalho é apresentar indicadores bibliométricos sobre o tema "sexualidade na adolescência" na base Scopus. Para tal, foi realizado um levantamento, na base Scopus, em julho de 2017 para identificação dos principais autores, instituições, periódicos e áreas que mais publicam a respeito do tema. Foi possível identificar que o Brasil é o 50 país que mais publica a respeito do tema, possuindo taxa de crescimento dos artigos da ordem de $20,8 \%$ ao ano, considerando os últimos 15 anos. As principais instituições que produzem artigos com autoria de brasileiros são USP, FIOCRUZ, UNICAMP e UFRGS. No mundo, todas as 10 principais instituições que mais publicam a respeito do tema estão nos Estados Unidos. As áreas que mais estudam a respeito do tema são a de medicina, ciências sociais e psicologia. Também foi possível identificar os principais autores brasileiros e mundiais, bem como os periódicos em que brasileiros e demais autores mais publicam a respeito do tema. Conclui-se que o tema sexualidade na adolescência tem cada vez mais despertado interesse da comunidade científica, sendo que o Brasil, além de estar relativamente bem situado entre os que mais publicam, também tem crescido suas taxas de crescimento de publicações referentes ao tema.

Palavras-chave: sexualidade na adolescência, bibliometria, produção científica

\section{ABSTRACT}

Adolescent sexuality is a problem that has been studied frequently due to the risk of unwanted and/or unplanned pregnancies, sexually transmitted diseases and sexual victimization. The aim of the present paper is to present bibliometric indicators on the theme "sexuality in adolescence" in the Scopus database. To this end, a survey was carried out, at Scopus base, in July 2017 to identify the main authors, institutions, journals and areas that publish the most about the topic. It was possible to identify that Brazil is the 5th country that publishes the most respect to the theme, with growth rates of articles in the order of $20.8 \%$ per year, considering the last 15 years. The main institutions that produce articles authored by Brazilians are USP, FIOCRUZ, UNICAMP and UFRGS. In the world, all the top 10 institutions that publish the most on the subject are in the United States. As areas that study more respect to the theme are medicine, social sciences and psychology. It was also possible to identify the main Brazilian and world authors, as well as journals in which Brazilians and other authors more published on the subject. Conclude that the topic of sexuality in adolescence is more aroused by the interest of the scientific community, and in Brazil, in addition to being relatively well placed among what is most publicized, the growth rates of publications related to the topic have also grown.

Keyword: sexuality in adolescence, bibliometrics, scientific production

Autor para correspondência: Eduardo Shimoda

E-mail: shimoda@ucam-campos.br

Av. Anita Peçanha, 100, Parque São Caetano, Campos dos Goytacazes - RJ - Telefone: 022-99828-8026 


\section{INTRODUÇÃO}

A sexualidade é um conjunto de importantes características biológicas, que sofre influências sociais e comportamentais durante todo o processo do desenvolvimento humano. As características fenotípicas sexuais podem ser visualizadas desde o período fetal como, por exemplo, a ereção peniana no sexo masculino e a lubrificação vaginal no sexo feminino, já presente nos primeiros dias de vida, demonstrando o potencial genotípico para o desenvolvimento da sexualidade. As sensações sexuais estão presentes durante todo o desenvolvimento da criança e são mais do que o prazer ligado ao aparelho genital, mas sim excitações ligadas a atividades inerentes do desenvolvimento, como a amamentação, que segundo Freud, proporciona um prazer que vai além da satisfação da necessidade fisiológica normal. Com o desenvolvimento físico característico, associado ao predomínio do pensamento abstrato e crítico, que o adolescente inicia um processo de autoconhecimento e independência que torna o ser humano apto a concretizar na adolescência, a sexualidade através do ato sexual propriamente dito. $\mathrm{O}$ aumento do interesse sexual coincide com o surgimento dos caracteres sexuais secundários. Este interesse é influenciado pelas profundas alterações hormonais deste período da vida e pelo contexto psicossocial (LEVIN, 1969).

Portanto o comportamento sexual de um indivíduo depende não só do seu componente biológico, definido pelas suas características genotípicas que vão orquestrar as repercussões fenotípicas moldadas pelo período do desenvolvimento em que se encontra, mas também e não menos importante pelo contexto familiar e sociocultural em que está inserido. Atualmente, a sociedade moderna tem produzido modelos de comportamento sexual confusos aos jovens, principalmente relacionado à época adequada do início das relações sexuais. Ao mesmo tempo em que promove a atividade sexual na adolescência como um fato natural, ainda persiste uma condenação moral e religiosa ao sexo antes do casamento. Este contexto dificulta o relacionamento, as atitudes e a adequada saúde sexual, no contexto biopsicossocial a estas moças e rapazes, que se encontram perdidos, sem um parâmetro social claro de comportamento sexual e com uma urgência biológica a ser satisfeita em idade precoce (FERREIRA et al., 2006).

Para a superação desses modelos sociais ambíguos sobre a sexualidade, faz-se necessário a promoção de uma educação sexual adequada, além de ações e serviços de saúde que auxiliem os adolescentes a lidar com a sexualidade com comportamentos de prevenção e de cuidado pessoal (CARIDADE, 1999). A Organização Mundial de Saúde (OMS, 2002), ao estabelecer os objetivos do trabalho com adolescentes, considera a adolescência uma fase de transição, entre o desenvolvimento biológico da infância até o amadurecimento sexual e reprodutivo; o desenvolvimento dos padrões cognitivos e emocionais da infância à idade adulta, respeitadas as particularidades culturais; e o desenvolvimento socioeconômico da pessoa em direção a sua relativa independência material, respeitando a organização social e econômica do grupo em que se encontra inserido.

Com o intuito de auxiliar na promoção da educação da sexualidade na adolescência, este tema tem sido estudado por diversos autores em diversos países e a mensuração de sua produtividade científica pode indicar o grau de investimento em pesquisas aplicadas a este tema, uma vez que a comunidade científica costuma responder aos problemas intrínsecos de uma localidade. Além disso, o compilar destas pesquisas, pode orientar a criação de diretrizes de uma política nacional de ensino no tema, uma vez que permite um diagnóstico das potencialidades de grupos e instituições (OLIVEIRA et al., 1992).

A bibliometria pode ser utilizada para avaliar a produtividade e a qualidade da pesquisa dos cientistas, por meio da medição com base nos números de publicações e citações dos diversos pesquisadores (MEIS, 1999). Os índices bibliométricos constituem uma eficiente forma de mensurar e diagnosticar os esforços de pesquisa e publicação relacionados a um determinado tema (TAGUE-SUTCKIFFE, 1992).

A Scopus é uma base de dados multidisciplinar, criada pela editora Elsevier em 2004, que cobre conteúdos publicados desde 1960. A base é atualizada diariamente, incluindo 21.000 títulos de mais de 5.000 editoras internacionais, 20.000 periódicos revisados por pares, 390 publicações comerciais, 370 séries de livros, 5,5 milhões de documentos de conferências, "Articles-in-Press" de mais de 3.850 periódicos e editoras, sendo fonte para bibliotecários, especialistas em informação, pesquisadores e editores (MESQUITA et al., 2006).

O objetivo do presente estudo é apresentar 
indicadores bibliométricos a respeito das publicações sobre "sexualidade na adolescência" na base Scopus.

\section{METODOLOGIA}

Foi realizada uma pesquisa utilizando a base de dados Scopus, para a coleta de dados as palavraschaves: "sexualidade" e "adolescência" em inglês (sexuality; adolescent, respectivamente). A busca foi realizada em julho de 2017, utilizando a opção de busca rápida, que resulta em publicações que tenham a palavra digitada no título, no resumo ou nas palavras-chaves, sem recorte temporal, ou seja, obtendo-se todas as informações independentemente do ano.

As sintaxes de busca utilizadas, com as expressões boolenas, foram:

Title-abs-key (sexuality and adolescent) and (limit-to (doctype, "ar")) and (limit-to (srctype, "j")).

Title-abs-key (sexuality and adolescent) and (limitto (doctype, "ar")) and (limit-to (srctype, "j")) and (limit-to (affilcountry, "brazil")).

Para fins de comparações com as demais publicações contidas na base de dados Scopus, foi realizada outra busca com o termo-chave "a", este representa toda produção cadastrada na base, partindo do princípio de que todas as produções contêm a letra "a". As sintaxes utilizadas foram:

- Title-abs-key (a) and (limit-to (doctype, "ar")) and (limit-to (srctype, "j"))

- Title-abs-key (a) and (limit-to (doctype, "ar")) and (limit-to (srctype, "j")) and (limit-to (affilcountry, "brazil")).

Para identificar informações relacionadas às análises quantitativas de: publicações, autores, instituições, países, áreas de interesse e veículos de comunicação; as buscas foram refinadas nos campos disponibilizados pela base, realizando a comparação dos dez primeiros contribuintes de cada caso.

Para análise temporal de crescimento anual foram geradas equações de regressão exponenciais da quantidade de publicações sobre: (a) todas as publicações da base Scopus, no Brasil e no mundo e, (b) sobre o tema específico (sexualidade na adolescência) no Brasil e no Mundo, em função do ano. $\mathrm{Na}$ análise temporal, utilizaram-se apenas os dados referentes aos anos de 2001 a 2015.

\section{RESULTADOS E DISCUSSÃO}

Os Estados Unidos constituem o principal país, relacionado a publicações na base Scopus, tanto no que se refere a produção científica geral, quanto ao tema "sexualidade na adolescência". No entanto, no caso específico do tema, o EUA é responsável por quase $50 \%$ das publicações mundiais, enquanto que considerando a publicação geral, os Estados Unidos possuem em torno de $27 \%$ dos artigos. A China é o segundo país que mais publica, independente do tema, mas não se encontra entre os 10 principais países que publicam a respeito de sexualidade na adolescência. Interessante documentar, que o Brasil é o 15 o país em termos de produção científica geral e, especificamente sobre o tema sexualidade na adolescência, ocupa o 5o lugar dentre os que mais publicam, o que demonstra certo preocupação dos pesquisadores brasileiros no sentido de estudar o tema (Figura 1).

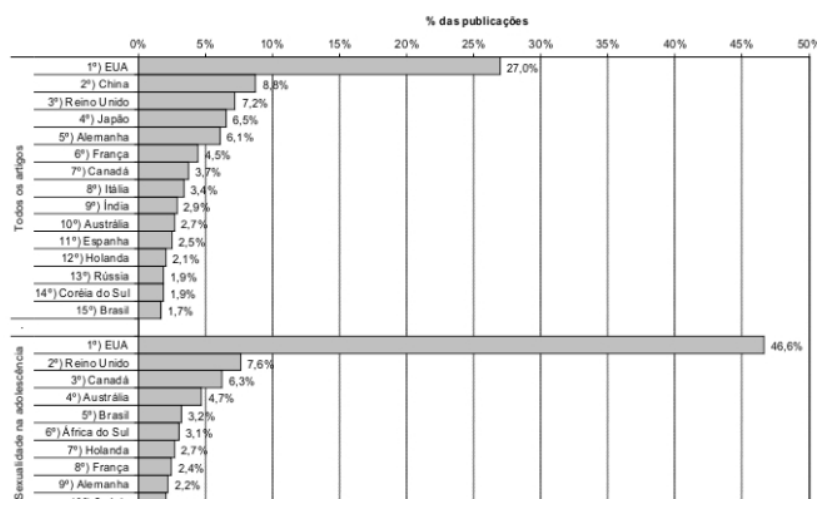

Figura 1: Países com mais publicações sobre todos os temas ou sobre o tema "sexualidade na adolescência" na base Scopus.

Os Estados Unidos predominam em relação a origem das instituições com mais publicações sobre o tema "sexualidade e adolescência", pois as 10 instituições que mais publicam sobre o tema, estão localizadas nos Estados Unidos. No Brasil, a USP é a universidade com maior número de publicações, seguida da Fiocruz, Unicamp e UFRGS. Percebe-se também, que no Brasil, existe uma relativa concentração das publicações oriundas de instituições localizadas na região Sudeste do país, composta por 8 das 10 que mais publicam (Figura 2). 


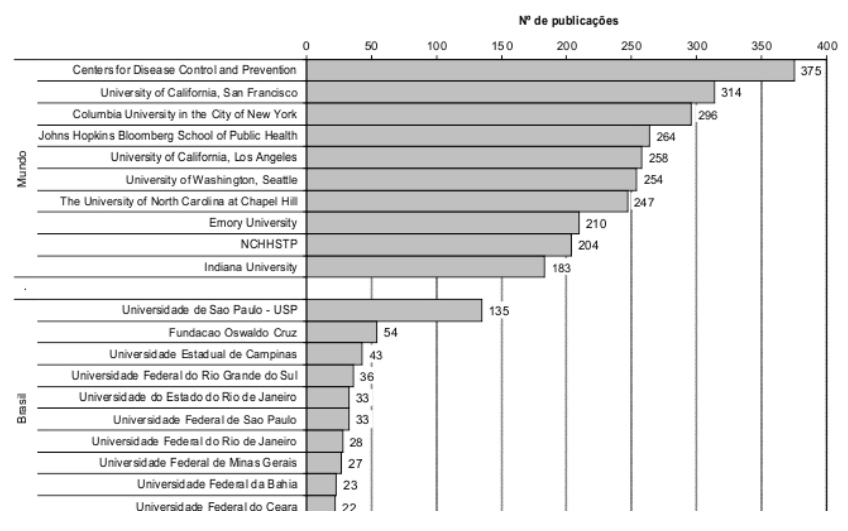

Figura 2 - As principais instituições que publicam sobre o tema "sexualidade na adolescência" no mundo e no Brasil.

Na Figura 3 é apresentado o número de publicações por ano a respeito de todos os temas e sobre o tema "sexualidade na adolescência" tanto no Brasil quanto no mundo.
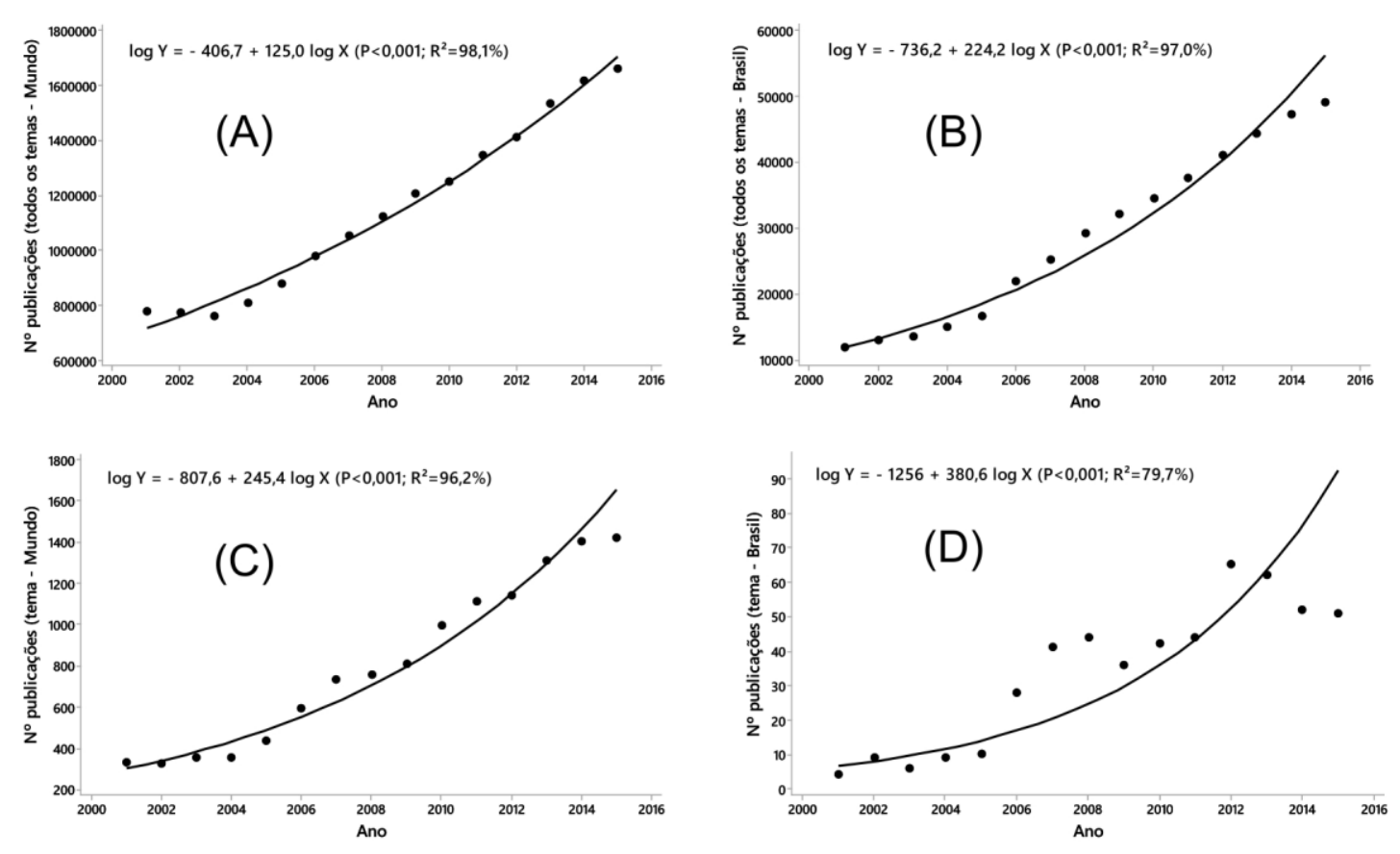

Figura 3 - Número de publicações por ano na base Scopus a respeito de: (a) todos os temas no mundo, (b) todos os temas no Brasil, (c) tema sexualidade na adolescência no mundo e, (d) tema sexualidade na adolescência no Brasil.

Com base nas equações de regressão do número de publicações por ano, foram calculadas as taxas de crescimento anual, cujos resultados são apresentados na Figura 4. Observa-se que o número de publicações sobre o tema "sexualidade na adolescência" quando comparada a todas as publicações na base Scopus, tem taxas de crescimento superiores, tanto no mundo $(13,0 \%$ e
$6,4 \%)$ quanto no Brasil (20,8\% e $11,8 \%)$. Isso que demonstra que o crescimento das publicações sobre o tema "sexualidade na adolescência" está mais acelerado do que a média de crescimento sobre o conjunto de publicações, independentemente do tema. De forma geral, o Brasil tem taxas de crescimento maiores do que a média mundial, tanto no conjunto das publicações da base Scopus $(11,8 \%$ e $6,4 \%)$ como quando se analisam as publicações específicas do tema "sexualidade na adolescência" (20,8\% e 13,0\%). É possível observar, portanto, que o tema estudado está despertando crescente interesse por parte dos pesquisadores, sendo que este comportamento se faz mais pronunciado no Brasil.

Os autores que apresentam as maiores publicações sobre o tema "sexualidade na adolescência" possuem entre 35 e 74 artigos na 
base Scopus. No Brasil, os autores que sobressaíram em relação ao número de publicações sobre este tema, possuem entre 7 e 24 artigos publicados na base Scopus. (Figura 5).

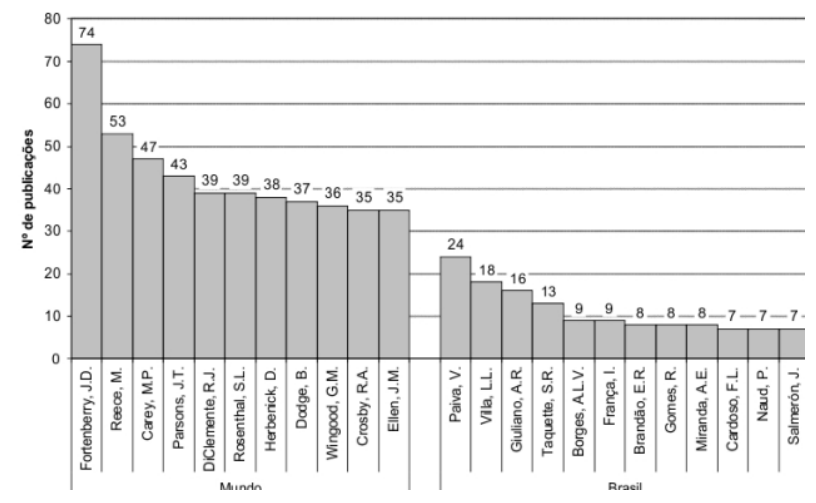

Figura 5 - Autores com maiores quantidades de publicações sobre o tema "sexualidade na adolescência" na base Scopus.

Em nível mundial, ("Journal Of Sex Reseach" e "Archives os Sexual Behavior"), são os periódicos mais contém artigos referentes ao tema "sexualidade na adolescência". No Brasil, os 3 principais periódicos são da área de saúde pública. Ainda, percebe-se predominância de periódicos de língua inglesa (os 10 periódicos que mais publicam) (Figura 6).

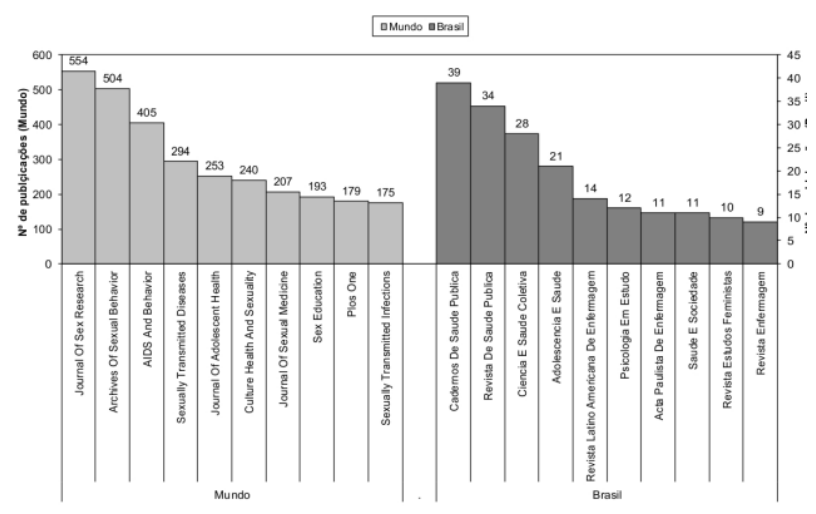

Figura 6 - Periódicos com mais publicações sobre o tema "sexualidade na adolescência".

Dentre as áreas em que as publicações relacionadas ao tema "sexualidade na adolescência" mais estão vinculadas, destacam-se as áreas de medicina, ciências sociais e psicologia, nesta ordem, tanto mundialmente quanto no Brasil conforme demonstrado na Figura 7. Interessante notar que, no mundo, existem maiores esforços das áreas de Ciências Sociais e Psicologia no estudo da sexualidade na adolescência, enquanto no Brasil, a área predominante é a medicina, tendo mais que o dobro das publicações que todas as outras áreas juntas. Nota-se também que, tanto no mundo quanto no Brasil, a Medicina predomina nas publicações sobre o tema, sendo seguido pelas Ciências Sociais e Psicologia, que exibem maiores índices publicações a nível mundial.

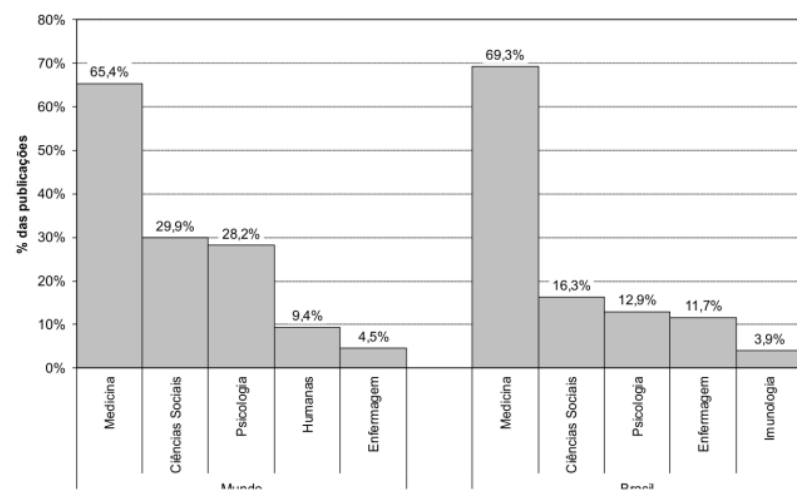

Figura 7 - Principais áreas de vinculação dos artigos publicados na base Scopus e relacionados ao tema "sexualidade na

\section{CONSIDERAÇÕES FINAIS}

Conclui-se que o número de publicações relacionadas ao tema "sexualidade na adolescência" tem apresentado taxas de crescimento mais aceleradas do que o verificado em média na base Scopus. O Brasil, além de ser o 5 o país que mais publica sobre o tema, tem aumentado de forma muito acelerada o número de publicações relativas ao tema. Também verificou-se que as principais instituições que mais publicam a respeito do tema é dos EUA, sendo que no Brasil, as publicações estão concentradas em instituições da região sudeste. As áreas que mais tem realizado esforços no sentido de estudar e pesquisar a respeito do tema são as de medicina, ciências sociais e psicologia, tanto no Brasil quanto no mundo. O crescimento do número de publicações sobre o tema, de certa forma demonstra a preocupação da comunidade científica sobre este assunto que pode comprometer toda estrutura familiar de uma sociedade, caso não seja tratado com a devida relevância que merece, através da aplicação de medidas político-econômico-sócioeducativas aos adolescentes e jovens a respeito de sua sexualidade.

Este trabalho tem sua importância relacionada ao mapeamento dos principais autores, instituições, áreas, periódicos em que se encontram 
publicações sobre o tema "sexualidade na adolescência", com o intuito de demonstrar quais são os principais centros de excelência de pesquisa na área e inferir de forma indireta os investimentos científicos sobre o tema.

\section{REFERÊNCIAS}

Levin M. Healthy sexual behavior. Pediatr Clin North Am. 1969; 16(2):329-32.

Ferreira V, Portella AP. Marco teórico e referencial: saúde sexual e saúde reprodutiva de adolescentes e jovens / Ministério da Saúde, Secretaria de Atenção à Saúde, Departamento de Ações Programáticas Estratégicas. Brasília: Editora do Ministério da Saúde; 2006.

Oliveira AC, Dórea JG, Domene SMA. Bibliometria na avaliação da produção científica da área de nutrição registrada no Cibran: período de 1984-1989. Ciência da Informação. 1992; 21(3): 239-42.

Tague-Sutckiffe J. An introduction to informetrics. Information Processing \& Management. 1992; $28(1): 1$-3.

Caridade A. O Adolescente e a Sexualidade. In: Brasil. Ministério da Saúde. Secretaria de Políticas de Saúde. Área de Saúde do Adolescente e do Jovem. Cadernos Juventude Saúde e Desenvolvimento. Brasília: Ministério da Saúde, 1999, p. 206-

212.

World Health Organization. Defining sexual health: report of a technical consultation on sexual health; 2002. p. 28-31 [cited 2011 Oct 10]. Available from: http://www.wpro.who.int

Meis L, Maia C, Lannes D, Machado RP. Uso de indicadores exige cautela. Folha de São Paulo, São Paulo, 12 set. 1999. Caderno Especial Ranking da Ciência, p. 7.

Mesquita R, Brambilla S, Laipelt RCF, Maia F, Vanz S, Caregnato S. Elaboração e aplicação de instrumentos para avaliação da base de dados Scopus. Perspectivas em Ciência da Informação. 2006; 11: 187-205. 